\title{
REPRESENTATION OF SEMIGROUPS AS SYSTEMS OF COMPACT CONVEX SETS
}

\author{
H. RATSCHEK AND G. SCHRÖDER
}

\begin{abstract}
ABSTRACr. Under Minkowski addition and scalar multiplication the system of all compact convex subsets of $R^{n}$ is an $R$-semigroup, i.e. a semigroup over the operator domain $R$ of real numbers with certain conditions for the operation of $R$ on the semigroup. Conversely, there is the question: When is an abstract $R$-semigroup isomorphic to a system $\subseteq$ of compact convex subsets of a suitable locally convex space? In this paper a necessary and sufficient condition for the existence of such a representation is given. This condition remains valid if, for the representing structures $\mathbb{S}$, systems of closed, bounded convex subsets with the closed Minkowski addition as addition are permitted. Finally, every $\boldsymbol{R}$-semigroup of compact convex subsets of any locally convex space is isomorphic to a system of rectangular parallelepipeds of some vector space.
\end{abstract}

1. Introduction. Let $\Re$ be the set of all compact convex subsets of $R^{n}$. Scalar multiplication and Minkowski addition are defined as usual:

$$
\begin{aligned}
\lambda K & =\{\lambda x: x \in K\} \quad \text { for } K \in \Re \text { and } \lambda \in R, \\
K_{1}+K_{2} & =\left\{x_{1}+x_{2}: x_{1} \in K_{1}, x_{2} \in K_{2}\right\} \quad \text { for } K_{1}, K_{2} \in \Re .
\end{aligned}
$$

It is known that $\Re$ forms an abelian semigroup with cancellation law and the field $R$ of real numbers acts as operator domain for $\Re$, satisfying the following rules for all $K, K_{1}, K_{2}$ in $\Re$ and all $\lambda, \mu$ in $R$ (cf. [5] and [2]):

$$
\begin{aligned}
\lambda\left(K_{1}+K_{2}\right) & =\lambda K_{1}+\lambda K_{2}, \\
\lambda K+\mu K & =(\lambda+\mu) K \quad \text { for } \lambda \mu \geqslant 0, \\
\lambda(\mu K) & =(\lambda \mu) K, \\
1 K & =K .
\end{aligned}
$$

Conversely, let $Q$ be an abelian semigroup over the operator domain $R$ satisfying the cancellation law and (2); then the question arises whether $Q$ is isomorphic to a system $\subseteq$ of compact convex subsets under the operations (1) in a not necessarily finite dimensional topological vector space. In this notice we shall give a necessary and sufficient condition for such a representation in a locally convex space.

An abelian semigroup $Q$ over $R$ with cancellation law will be called an $R$-semigroup with c.l. if the conditions (2) are satisfied. Note that $Q$ must contain a zero element $\theta$ such that $0 K=\theta$ for all $K$ in $Q$. If the property

Received by the editors September 2, 1976.

AMS (MOS) subject classifications (1970). Primary 20M30; Secondary 52A05.

Key words and phrases. Representation of semigroups, systems of compact convex sets. 


$$
(\lambda+\mu) K=\lambda K+\mu K \text { for all } \lambda, \mu \in R \text { and } K \in Q
$$

holds in an $R$-semigroup $Q$ with c.l., then $Q$ is a vector space over $R$. We write $-K$ instead of $(-1) K$. An element $K \in Q$ is said to be symmetric if $K=-K$ and, likewise, $Q$ if all elements of $Q$ are symmetric. The set $Q_{s}$ of all symmetric elements of $Q$ is an $R$-subsemigroup of $Q$. In this connection we refer to a paper of Rabinovich [4]. There, for a Riesz space $L$, necessary and sufficient conditions are given for the existence of a system $\mathcal{S}$ of convex sets such that $L$ is isomorphic to the system $\mathfrak{T}=(\mathfrak{S} \times \mathfrak{S}) / \rho$. Here $\rho$ is a congruence in the sense of [5] and the partial order on $\mathfrak{T}$ is induced by the inclusion in $\subseteq$.

2. Reduction to the symmetric case. The following Lemma shows that the question for representability of an $R$-semigroup with c.l. as a system of compact convex sets can be reduced to symmetric $R$-semigroups.

LEMMA. Each $R$-semigroup $Q$ with c.l. is isomorphic to a subdirect product of a vector space and $Q_{s}$, the subsemigroup of all symmetric elements of $Q$.

Proof. We remember the following definition and theorem, cf. Cohn [1]: If $Q_{1}$ and $Q_{2}$ are $R$-semigroups, then an $R$-subsemigroup $Q_{0}$ of the direct product $Q_{1} \times Q_{2}$, which is such that $Q_{1}$ and $Q_{2}$ are homomorphic images of $Q_{0}$, is said to be a subdirect product of $Q_{1}$ and $Q_{2}$. If $Q_{0}$ is an $R$-semigroup with congruences $\rho_{1}$ and $\rho_{2}$ such that $\rho_{1} \cap \rho_{2}=\Delta$ with $\Delta=\{(K, K): K \in$ $\left.Q_{0}\right\}$, then $Q_{0}$ is isomorphic to a subdirect product of $Q_{0} / \rho_{1}$ and $Q_{0} / \rho_{2}$.

For proving the Lemma we chose suitable congruences for $Q$. Let the congruences $\sigma$ and $\tau$ be defined by

$X \sigma Y$ if there exist $S, T \in Q_{s}$ such that $X+S=T+Y$.

$X \tau Y$ if $X-X=Y-Y$.

Then $Q / \sigma$ is the largest vector space over $R$ being a homomorphic image of $Q$, cf. [2]. Next we show $Q_{s} \simeq Q / \tau$. A corresponding isomorphism is given by the restriction $p \mid Q_{s}$ of the natural projection $p: Q \rightarrow Q / \tau$ to $Q_{s}$.

We see that $p \mid Q_{s}$ is injective: Let $S, T \in Q_{s}$ and $p(S)=p(T)$, that is $S-S=T-T$ by definition of $\tau$. Because of the symmetry of $S$ and $T$ this equation leads to $2 S=2 T$ and $S=T$. We see that $p \mid Q_{s}: Q_{s} \rightarrow Q / \tau$ is onto, because every congruence class $p(X)$ with $X \in Q$ contains the symmetric element $S=\frac{1}{2}(X-X)$ and we have $p(X)=p(S)$ with $S \in Q_{s}$. We see that $\sigma \cap \tau=\Delta$ is satisfied: Trivially, we have $\Delta \subset \sigma$ and $\Delta \subset \tau$. Now, let $(X$, $Y) \in \sigma \cap \tau$; this means that the equations $X+S=Y+T$ and $X-X=Y$

- $Y$ hold for some $S, T \in Q_{s}$. Further,

$Y-Y+S=X-X+S=Y+T-X$, respectively.

$S-Y=T-X$ and $Y+S=X+T$ by the cancellation law. Accordingly, from

$$
Y+S+S=X+S+T=Y+T+T
$$

we get $S=T$ and forward $X=Y$, that is $(X, Y) \in \Delta$. This proves the Lemma. 
From the Lemma we conclude that the $R$-semigroup $Q$ with c.l. is representable as a system of compact convex subsets of some locally convex space if and only if the $R$-subsemigroup $Q_{s}$ is representable as such a system: If $Q$ is isomorphic to a system $\subseteq$ of compact convex sets, then $Q_{s} \subset Q$ is isomorphic to some subsystem of $\mathfrak{S}$. Conversely, let, for the $R$-semigroup $Q$ with c.l., the $R$-subsemigroup $Q_{s}$ be isomorphic to a system $\Im_{s}$ of compact convex subsets of a locally convex space $L$. By the Lemma, $Q$ is isomorphic to a subdirect product of a vector space $V$ and $Q_{s}$. The space $V$ can be equipped with any locally convex topology such that the product space $L \times V$ is locally convex too. A system of compact convex subsets of $L \times V$ that is closed with respect to scalar multiplication and Minkowski addition is given by

$$
\mathfrak{I}=\left\{(K,\{Z\}): K \in \mathfrak{S}_{s}, Z \in V\right\} .
$$

Finally, from $\mathfrak{I} \simeq Q_{s} \times V$ we conclude that $Q$ is embeddable into $\mathfrak{T}$.

3. Representation theorems. We shall now give a necessary and sufficient condition for a representation of a symmetric $R$-semigroup $Q$ with c.l. as a system $\mathfrak{S}$ of compact convex sets in a locally convex space with scalar multiplication and Minkowski addition (cf. (1)) as operations on $\mathfrak{S}$. A real functional $f$ on $Q$ is said to be positive linear if $f$ is additive and positive homogeneous (i.e. $f(\lambda X+\mu Y)=|\lambda| f(X)+|\mu| f(Y)$ for $\lambda, \mu \in R$ and $X$, $Y \in Q$ ) and if the values of $f$ are nonnegative. Let $Q^{+}$be the set of all positive linear functionals on $Q$. Then $Q^{+}$is a symmetric $R$-semigroup with c.l. if addition and multiplication with nonnegative scalars are defined pointwise and if $\lambda f=(-\lambda) f$ for $f \in Q^{+}$and $\lambda<0$. We note down the following separation condition for symmetric $R$-semigroups:

(P) For distinct elements $X, Y \in Q$ there exists an $f \in Q^{+}$such that $f(X) \neq f(Y)$.

THEOREM 1. Let $Q$ be a symmetric $R$-semigroup with $c . l$ satisfying condition (P). Then there exists a system $\subseteq$ of compact convex sets in a locally convex space $L$ such that $Q$ and $\subseteq$ are isomorphic.

Proof. Let $L=R^{\alpha}$ be the product space with respect to the natural topology on $R$ where $\alpha$ is the cardinality of $Q^{+}$. Let $\Re$ be the system of all compact convex sets in $L$. We construct a monomorphism $\iota: Q \rightarrow \Re$, as follows. Every $X \in Q$ can be regarded as a positive linear functional on $Q^{+}$ defined by $X(f)=f(X)$ for $f \in Q^{+}$. Considering the family $([-X(f)$, $X(f)])_{f \in Q^{+}}$, we define $\iota(X)=\Pi([-X(f), X(f)])_{f \in Q^{+}}$. Then $\iota(X)$ is a compact convex set in $R^{\alpha}$. The system $\subseteq=\{\iota(X): X \in Q\}$ is closed under the operations (1), and $\iota$ is a homomorphism. Clearly, $\iota$ is injective, because by (P) there exists an $f \in Q^{+}$with $f(X) \neq f(Y)$ for $X \neq Y$, which entails $\iota(X) \neq \iota(Y)$ since $f(X), f(Y) \geqslant 0$.

THEOREM 2. If a symmetric $R$-semigroup $Q$ with c.l. is isomorphic to a system S of compact convex sets in a locally convex space $L$ then $(\mathrm{P})$ holds in $Q$. 
Proof. For $Z \in Q$, the corresponding convex set in the system $\mathbb{S}$ is denoted by $Z^{\prime}$. Let $L^{*}$ be the dual space of $L$ and $H_{Z}: L^{*} \rightarrow R$ be the supporting function of $Z^{\prime}$, i.e. $H_{Z}(\varphi)=\sup \left\{\varphi(x): x \in Z^{\prime}\right\}$.

Now it follows from [3] that for $X \neq Y$ there exists a $\varphi \in L^{*}$ with

$$
H_{X}(\varphi) \neq H_{Y}(\varphi) \text {. }
$$

If the functional $f$ on $Q$ is defined by $f(Z)=H_{Z}(\varphi)$ then we have $f(X) \neq$ $f(Y)$ by (3). It remains to show that $f \in Q^{+}$. The values of $f$ are nonnegative since $H_{Z}(\varphi) \geqslant 0$, following from $0 \in Z^{\prime}$ and $\varphi(0)=0$. Using

$$
f\left(\lambda Z_{1}+\mu Z_{2}\right)=\lambda f\left(Z_{1}\right)+\mu f\left(Z_{2}\right) \text { for } \lambda, \mu \geqslant 0,
$$

cf. [3], and $f(Z)=f(-Z)$ for $Z, Z_{1}, Z_{2} \in Q$, we obtain $f \in Q^{+}$.

REMARK 1 . Theorem 1 can be stated without making use of a topology as can be seen from its proof. Therefore, under the assumption of Theorem $1, Q$ is isomorphic to a system of parallelepipeds $\Pi\left(\left[-a_{i}, a_{i}\right]\right)_{i \in I}$ in the vector space $R^{I}$, where $I$ is a suitable index set.

REMARK 2. Theorem 2 can be reformulated when using closed, bounded convex sets instead of compact convex sets. Since the sum of closed sets under Minkowski addition need not be closed, we replace it by the closed Minkowski addition

$$
K_{1}+K_{2}=\overline{\left\{x_{1}+x_{2}: x_{1} \in K_{1}, x_{2} \in K_{2}\right\}} .
$$

Now (3) and (4) with respect to this addition remain valid for closed, bounded convex subsets of a locally convex space $L$ (cf. [3]), hence also Theorem 2 using this addition and systems of those sets. As a consequence, every symmetric $R$-semigroup $S$ of closed, bounded convex sets in a locally convex space $L$ is isomorphic to a system of parallelepipeds in some vector space.

Remark 3. If (P) is not valid in a symmetric $R$-semigroup $Q$, then by Theorem 2 there exists no representation with a system of compact convex subsets. So we look for a representation using convex subsets only; hence we do not need any topology and shall work with vector spaces only. Statement: A symmetric $R$-semigroup $Q$ is isomorphic to a system $\subseteq$ of convex subsets of a vector space $L$ if and only if $A+B=\theta$ implies $A=B=\theta$ for all $A$, $B \in Q$. The necessity of this condition follows from properties of point sets in vector spaces. The condition is also sufficient: A theorem of Rảdström [5] implies that there is an additive and positive homogeneous bijection such that $Q_{s}$ (cf. \$2) is mapped onto a proper convex cone $C$ with vertex 0 in a vector space $L$. Then there exists a hypercone $H \supset C \backslash\{0\}$, also with vertex 0 . If we take $H \cup\{0\}$ as positive cone, the space $L$ is a vector lattice. If $\hat{X} \in C$ is the image of $X \in Q_{s}$ under the above bijection, then by $X \mapsto[-\hat{X}, \hat{X}]$ we get a monomorphism of $Q_{s}$ into the system $\subseteq$ of all symmetric order intervals of $L$.

\section{REFERENCES}

1. P. M. Cohn, Universal algebra, Harper and Row, New York, 1965. MR 31 \#224. 
2. G. Ewald and G. C. Shephard, Normed vector spaces consisting of classes of convex sets, Math. Z. 91 (1960), 1-19.

3. L. Hörmander, Sur la fonction d'appui des ensembles convexes dans un espace localement conoexe, Ark. Mat. 3 (1955), 181-186.

4. M. G. Rabinovich, Some classes of spaces of convex sets and their extensions, Sibirsk. Mat. $\grave{Z}$. 8 (1967), 1405-1415.

5. H. Ràdström, An embedding theorem for spaces of convex sets, Proc. Amer. Math. Soc. 3 (1952), 165-169.

Mathematisches InstTtut, Universttät Düsseldorf, Düsseldorf, Germany 